DCPT $/ 05 / 122$

IPPP/05/61

\title{
Scalars in the hadron world: the Higgs sector of the strong interaction
}

\author{
M.R. Pennington \\ Institute for Particle Physics Phenomenology, \\ Durham University, Durham, DH1 3LE, U.K.
}

\begin{abstract}
Scalar mesons are a key expression of the strong physics regime of QCD and the role condensates, particularly $\langle q \bar{q}\rangle$, play in breaking chiral symmetry. What new insights have been provided by recent experiments on $D, D_{s}$ and $J / \psi$ decays to light hadrons is discussed. We need to establish whether all the claimed scalars $\sigma, \kappa, f_{0}(1370)$, etc., really exist and with what parameters before we can meaningfully speculate further about which is transiently $\bar{q} q, \overline{q q} q q$, multi-meson molecule or largely glue.
\end{abstract}

Invited talk at the International Conference on QCD and Hadronic Physics, Beijing, June 2005. 


\section{Structure of the QCD vacuum}

This meeting celebrates QCD as the theory of the strong interactions. Many of its successes over the past 30 years stem from its remarkable property of asymptotic freedom. This means that at short distances we can use perturbation theory to describe hard scattering processes, make predictions and find they agree with experiment. But the bulk of hadronic phenomena involve interactions typically over the size of a proton and over such distances the interactions are strong. For these, QCD is far more difficult to solve and so we have to use a mixture of modelling, approximations and guidance from experiment. The strong coupling regime of QCD is responsible for the spectrum of hadrons, which in turn is intimately related to confinement and for light hadrons to the property of chiral symmetry breaking. These are the topics this talk encompasses.

QCD is rooted in the spectrum of hadrons and the relation of this to the quark model. Ideas we will question here. Nevertheless, the bulk of hadrons do fit into quark model multiplets. For $\bar{q} q$ states, the template is that for vector mesons formed by combining a quark and an antiquark in a spin-one $(S=1)$ system with no orbital angular momentum $(L=0)$ between them. Then with three flavours of quark, we expect 9 vector mesons. Nature tells us that the states in the middle of the multiplet with zero third component of isospin, the $\rho, \omega$ and $\phi$ are states of definite quark flavour. This we infer from their pattern of decays and their masses. Almost all mesons fit into such ideally mixed multiplets. However, it is well known that there are far more scalars than can fit into one such multiplet with $S=L=1$. We have the $f_{0}(400-1200), f_{0}(980), f_{0}(1370), f_{0}(1500)$, $f_{0}(1710), K_{0}^{*}(1430), a_{0}(980), a_{0}(1430)$ and perhaps even others [1]. Why does it matter that these don't fit our quark model template? Why indeed is there a special talk at this conference on the scalars? This is because the scalars are special. They are the Higgs sector of the strong interaction and directly reflect the structure of the QCD vacuum.

In QED, the vacuum, through which an electron in an atom moves, is filled with electronpositron pairs. The effect of these can be calculated in perturbation theory and so determine the fine structure of atomic spectra. Because of asymptotic freedom, the QCD vacuum appears similar over short distances. Quarks move through a sea of $\bar{q} q$ pairs and a shoal of gluons, but, so strong are the interactions between quarks and gluons over long distances, these change the nature of the vacuum. The long range correlations form condensates of quarks, antiquarks and gluons.

The symmetries of the hadron world are a reflection of QCD. While isospin symmetry follows from the near equality of up and down quark masses, it is their current masses that enter the QCD Lagrangian. These are only a few $\mathrm{MeV}$ and so much less than the natural scale of QCD, viz. $\Lambda_{Q C D}$, which is $100-200 \mathrm{MeV}$. Thus to a good approximation the up and down quarks can be regarded as massless. The QCD Lagrangian then has a bigger symmetry. Left-handed ups and downs can be interchanged independently of those spinning right-handedly and vice-versa. Consequently, the QCD Lagrangian has an $S U(2) \otimes S U(2)$ symmetry, and in as much as strange quarks are light, it has a more approximate $S U(3) \otimes S U(3)$ symmetry. These symmetries would be mirrored in hadron interactions too, but we do not see them. Scalars and pseudoscalars, vectors and axial-vectors do not have simply related interactions and masses. This is because the symmetry is broken. Nambu proposed a model [2] of this even before QCD was discovered. If the symmetric potential generated by scalar and pseudoscalar interactions was not like a bowl, but Mexican hat shaped, then nature would spontaneously break the symmetry. Let us call the scalar $\sigma$ and identify the pseudoscalar 
with the $\pi$. The scalar has a non-zero vacuum expectation value. The physical particles that correspond to the quantum fluctuations about the minimum of the potential (the vacuum) feel no resistance in the $\pi$ direction and the pions are massless, while the oscillations in the $\sigma$ direction go up and down the parabolic sides of the hat and so have mass [2, 3]. This spontaneous breaking of chiral symmetry is just like the magnetisation of a ferromagnet. The pions, the lightest of all hadrons, are the Goldstone bosons, while the scalars are the Higgs sector. Their mass gives mass to all light hadrons. At the QCD level, the chiral symmetry is dynamically broken by the formation of condensates.

In the last few years we have learnt that this breaking is dominated by the non-zero value of the $\bar{q} q$ condensate with a scale of $\sim-(250 \mathrm{MeV})^{3}$. We learn this phenomenologically using QCD sum-rules [3], theoretically from calculations of quark mass functions in the chiral limit which are made possible by using the Schwinger-Dyson equations in the continuum [3], and most recently experimentally by accurate determinations of low energy $\pi \pi$ scattering from $K_{e 4}$ decay [4] and studies of pionium [5]. These confirm that the expansion of the pion mass in terms of quark mass is dominated by the very first term [6], as in the Gell-Mann-Oakes-Renner relation [7]:

$$
m_{\pi}^{2} f_{\pi}^{2}=-\left(m_{u}+m_{d}\right)\langle O|\bar{q} q| O\rangle+O\left(m_{q}^{2}\right)
$$

where $f_{\pi}$ is the pion decay constant. This dominance by the first term tells us that Nambu's ferromagnetic analogy is very close to what happens in the real world. Consequently, it is natural to ask what is the scalar field, whose non-zero vacuum expectation value breaks the chiral symmetry and whose mass reflects the constituent mass of light quarks? What is the chiral partner of the pion, and to a more approximate extent what is the chiral partner of the kaon? What are the $\sigma$ and the $\kappa$ ? Are they just one field or a collection of particles, $f_{0}$ 's and $K_{0}^{*}$ 's, seen in experiment?

\section{Scalars in scattering experiments}

Let us first briefly review what we know about the scalars from the classic meson-meson scattering experiments and then we will turn to what the more recent decay results tell us. The easiest states to identify unambiguously are the strange ones. These we learn about from high energy $K \pi$ production in $K p$ collisions, which at small momentum transfers are dominated by one pion exchange. From the famous LASS experiment [8] of twenty years ago, we see that $K \pi$ scattering is dominated by the spin- $1 K^{*}(892)$ and the spin- $2 K_{2}^{*}(1430)$ each seen in the appropriate partial wave with the rapid phase variation expected of a resonating wave. Under these the spin-0 wave has a broad bump rising to the unitarity limit with the slower phase variation expected of a state of $250 \mathrm{MeV}$ width. This is the $K_{0}^{*}(1430)$. These are the $I=1 / 2$ scalar mesons.

The $I=1$ states are found by partial wave analysing channels like $\pi^{-} p \rightarrow\left(\pi^{0} \eta\right) n$ studied by GAMS [9]. Again this process is dominated at small momentum transfers by pion exchange and reveals two states the $a_{0}(980)$ and $a_{0}(1430)$. The fact that there are two low mass isotriplets, may suggest that there should be another pair of isodoublets too. The LASS results on $K^{-} \pi^{+}$scattering start at $825 \mathrm{MeV}, 200 \mathrm{MeV}$ above threshold, and there has been intense speculation that there may be another scalar state, the $\kappa$, closer to threshold. We will discuss this possibility shortly. 


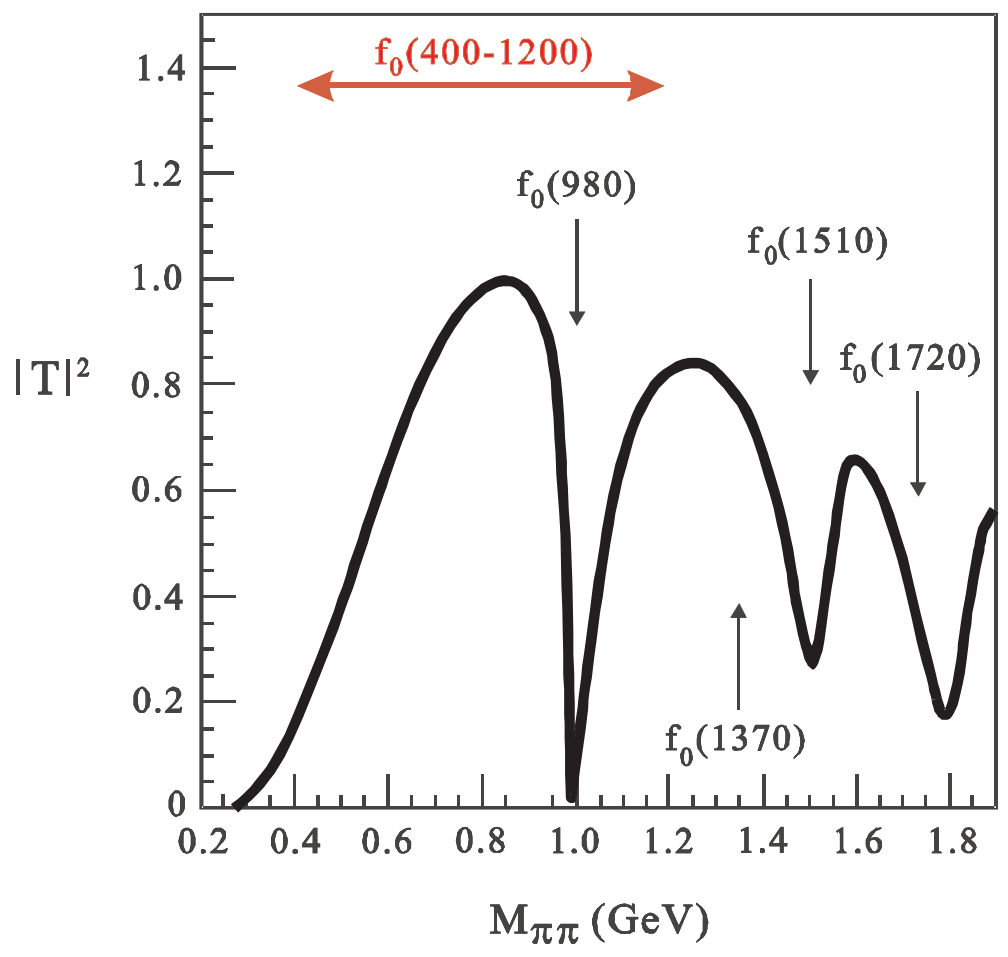

Figure 1: A sketch of the square of the modulus of the $I=0 \pi \pi S$-wave amplitude, from Zou ${ }^{10}$.

The $I=0$ channel is studied in $\pi \pi$ scattering. The $J=0$ cross-section shown in Fig. 1 reveals a series of peaks and dips [10], none of which is describable by any simple Breit-Wigner form. The first dip is strongly correlated with the onset of the $\pi \pi \rightarrow \bar{K} K$ channel and marks the $f_{0}(980)$. From Fig. 1 we see many overlapping isoscalar states. Any channel claiming to see any of these must include a description of all of them (though they may well appear with different strengths in different processes). They are inextricably mixed with each other and with the thresholds to which they couple. One cannot arbitrarily pick and choose and describe one or two with simple Breit-Wigner forms and ignore the others.

Fig. 1 indicates 5 low mass states. These could populate two nonets and still leave one that might be a glueball. Before speculating about the nature of such states, let us first illustrate why we need precision results to be certain that they all exist as poles in the complex energy plane. This need is readily understood if we consider the complex $s$-plane for $K \pi$ scattering. The amplitude (whether the forward full amplitude, or individual partial wave amplitudes) has a right hand cut produced by direct channel dynamics, and a left hand cut generated by crossed channel exchanges. In Fig. 2 is shown this plane. Along the top of the right hand cut, the region accessible in the LASS experiment is delineated. The determination of poles requires an analytic continuation, which is only accurately possible near to this region. Thus we can find the $K^{*}(892), K_{2}^{*}(1430)$ and even the wider $K_{0}^{*}(1430)$, but whether there is a low mass $\kappa$ or not depends on continuing experimental results into "unknown" territory. There is certainly no such pole with $\operatorname{Re} s>0.7 \mathrm{GeV}^{2}$ as shown in Ref. 11. However, different parametrisations of the data do yield a pole [1] deep in the complex plane below $\operatorname{Re} s<0.6 \mathrm{GeV}^{2}$. But this continuation enters regions just as close to the left hand cut as to where we have scattering data. Consequently, parametrisations that do not have the 


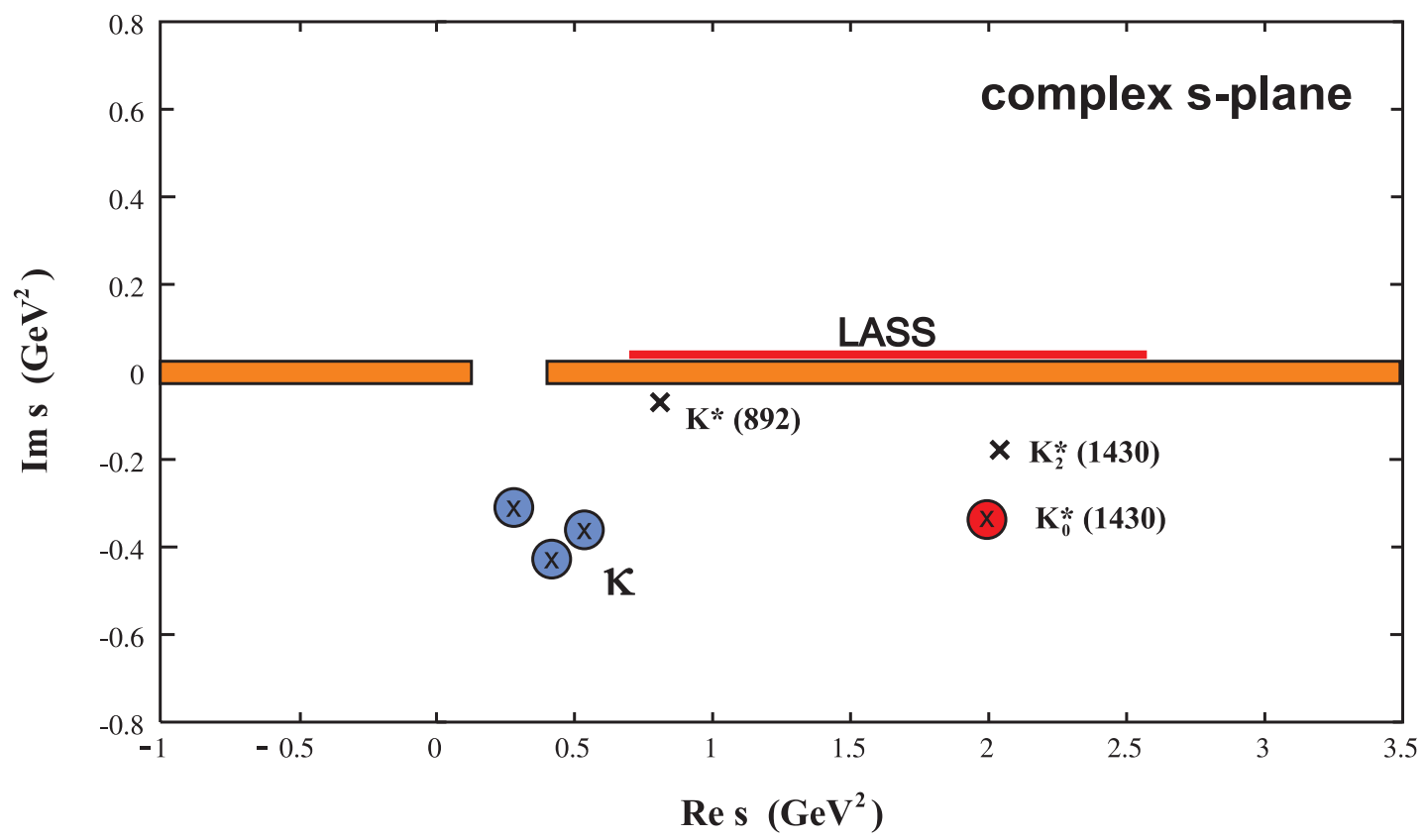

Figure 2: The complex $s$-plane for $K \pi$ scattering, showing the cut structure, and the nearby poles on the unphysical sheet. The possible positions of the $\kappa$-pole are from the listing in Ref. 1.

correct left hand cut analyticity, like simple $s$-channel Breit-Wigner forms, are highly suspect. The use of chiral perturbation theory to constrain the continuation is clearly an improvement, but the uncertainties in how to sum all orders are so large that we cannot be sure that the pole is not really much deeper. It is then an open question whether such a pole is meaningfully present.

\section{Scalars in decays and in the complex energy plane}

Fresh light is shed on the nature of the scalars by heavy flavour decay results - on $J / \psi$ and $D$-decays in particular. Let us start with the semileptonic decay $D^{+} \rightarrow\left(K^{-} \pi^{+}\right) \mu^{+} v_{\mu}$, which FOCUS [12] has measured in the 700-900 MeV $K \pi$ mass range. In this region the strong interaction component is dominated by the $K^{*}(892)$. If this was all there was then there would be no forwardbackward asymmetry in the $K \pi$ rest frame. However, there is a strong asymmetry, shown in Fig. 3. This tells us that in addition to the $P$-wave there is an $S$-wave $K \pi$ interaction with phase of about $45^{\circ}$. This is just what we would expect from a dominant $I=1 / 2$ final state interaction with phase given by the LASS results [8] as required by Watson's theorem. The phase is not $90^{\circ}$ (Fig. 3), which a Breit-Wigner for a low mass $\kappa$ would suggest. So far $D_{l 4}$ decay has only been studied in the 700-900 MeV region: the undetected neutrino causes the difficulty. Nevertheless, higher statistics from $B$-factories have the potential to allow the extraction of the $S$-wave $K \pi$ phase in different charge channels down towards threshold. This would provide a key piece of the jigsaw puzzle of low energy hadron dynamics. 

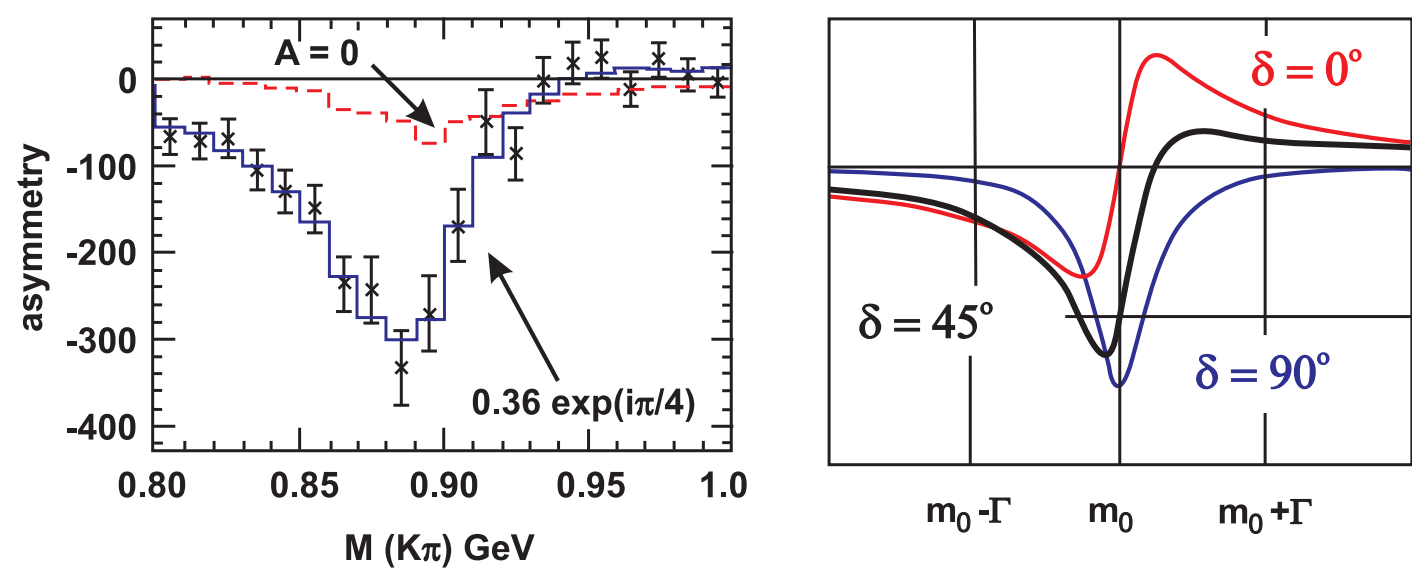

$m_{0}-\Gamma \quad m_{0} \quad m_{0}+\Gamma$

Figure 3: Asymmetry distribution in $K \pi$ invariant mass from the FOCUS experiment ${ }^{12}$. In the left hand figure the dashed line represents a simulation with no interfering $S$-wave. The solid line is with an $S$-wave of constant magnitude and phase of $\pi / 4$. The plot on the right shows how the asymmetry (defined in Ref. 12) changes for three different choices of $S$-wave phase.

We now turn to the potentially more complicated multi-hadron $D$-decays such as $D^{+} \rightarrow K^{-} \pi^{+} \pi^{+}$. With well established resonances dominating the $K^{-} \pi^{+}$interaction with spin-1 and spin-2, one can use knowledge of these to determine the $S$-wave amplitude, both magnitude and phase in $K \pi$ mass bins across the Dalitz plot. These have been determined from the E791 data by Brian Meadows [13]. These show a variation of phase that is not wholly attributable to the $I=1 / 2 S$-wave. As illustrated by Laura Edera and I [14] one can use the way the magnitude and phase change through the region of elastic unitarity to separate the $I=1 / 2$ and $I=3 / 2 S$-waves. An important feature of these results is that they already provide phase information down to $K \pi$ threshold, even without $D_{\ell 4}$ results. While the E791 data are not yet of sufficient precision to determine the phase within a fraction of a degree, higher statistics results from BaBar hold out the prospect of being able to constrain the $S$-wave amplitude down towards threshold at least as well as the analytic continuation of the LASS results [8] allows and hence help to determine whether a $\kappa$ resonance really exists as a pole in the complex energy plane or not.

In the $I=0$ sector it has long been known that the shape of the $\pi \pi$ mass distribution reflects the production mechanism. In Fig. 1, we have already seen the shape of the cross-section in $\pi \pi \rightarrow \pi \pi$ scattering with its broad low mass enhancement and deep dip at $1 \mathrm{GeV}$. For central $\pi \pi$ production in pp collisions at the ISR or $\pi^{-} p$ interactions at WA102, the $\pi \pi$ mass distribution is more peaked just above threshold because in this process there is no Adler zero. The reaction is essentially Pomeron-Pomeron scattering to $\pi \pi$ which is controlled by $\pi$ exchange as discussed long ago in Ref. 15. There the $f_{0}(980)$ appears as a shoulder. In contrast, in $J / \psi \rightarrow \phi(\pi \pi)$ and $D_{s} \rightarrow \pi(\pi \pi)$, where hidden strangeness is the source of the $\pi \pi$ system, the $f_{0}(980)$ appears as a striking peak. This preference for coupling to $\bar{s} s$, or equivalently virtual $\bar{K} K$, would in a conventional quark model multiplet indicate that the $f_{0}(980)$ is predominantly the $\bar{s} s$ state. However, as is well known, it is then difficult to understand how it can be degenerate in mass with the $a_{0}(980)$, which would have no strange quarks. This, of course, begs a very basic question. When are hadrons and their underlying quark model states closely identified? 
We know the $\phi$ meson is not just $\bar{s} s$, but contains within its Fock space contributions from $\bar{K} K$ and $\rho \pi$. It is through these components that it decays. However, these multi-meson components are small. They affect the parameters of the vector meson little. Consequently, we identify the $\phi$ with $\bar{s} s$ and the $\rho^{+}$, for instance, with $u \bar{d}$, even though they contain $\bar{K} K$ and $\pi \pi$ components, respectively. Vector states coupling to two pseudoscalars require $P$-wave interactions that are naturally suppressed near threshold. The fact that additional quark loops contribute little is, of course, totally in keeping with the $1 / N_{c}$ expansion, which would make these loops higher order. Unquenching is unimportant. In contrast, for the scalars, like the $f_{0}$ or $a_{0}^{+}$, even if they are seeded by $\bar{s} s$ and $u \bar{d}$, respectively, quark loops do matter. They drastically affect the states and give the $f_{0}$ and $a_{0}$ each a $\bar{K} K$ component [16, 17] that is as big as $\sim 40 \%$. Thus whatever their intrinsic makeup they have a significant long range component like a $\bar{K} K$ molecule. Unquenching is important in the scalar sector. There is no $1 / N_{c}$ suppression. Indeed, this means the OZI rule does not hold here. Not only do $\bar{s} s$ and $\bar{u} u, \bar{d} d$ communicate, but the presence of these resonances near $1 \mathrm{GeV}$ even enhances this communication [18]. This must be a clue to the flavour structure of the QCD vacuum.

Counting states determines whether the inevitable $\bar{K} K$ nature of the $f_{0}, a_{0}(980)$ is the result of an intrinsically molecular structure as claimed by Weinstein and Isgur [16], or whether they are just two examples of primarily 4 quark configurations. In the latter case there would be two low mass scalar multiplets, one of $\bar{q} q$ and the other $\overline{q q} q q$, as proposed by Jaffe [19], by Schechter and collaborators [20] and more recently by Maiani et al. [21], amongst others. The idea of systems built of scalar diquarks of different flavours, like $[u d],[u s]$ and $[d s]$, has recently received renewed interest with the many discussions of the $\Theta^{+}(1540)$ the putative pentaquark baryon. This has highlighted the possibility of a nonet of tetraquark states made of these three light types of diquarks. While in a conventional $\bar{q} q$ nonet, the $\bar{s} s$ state is the heaviest, in a tetraquark multiplet there is a degenerate isotriplet as well as an isosinglet built of $[\overline{n s}][n s]$ with $n=u, d$. This would naturally explain the degeneracy of the $a_{0}(980)$ and $f_{0}(980)$. In such a modelling, the $\bar{q} q$ nonet is heavier and would incorporate the $K_{0}^{*}(1430), a_{0}(1430)$, and two of the $f_{0}(1370), f_{0}(1500)$ and $f_{0}(1710)$ leaving one to be a glueball. Different schemes favour one or the other to be largely gluish as we have heard at this meeting [22]. The lighter tetraquark multiplet then needs strange isodoublets, the $\kappa$, and lightest of all a $[\overline{u d}][u d]$ ( $f_{0}$ or) $\sigma$. However seductive this picture, further discussion is not fruitful until we have established the existence of the complete set of states: in particular $\sigma, \kappa$ and $f_{0}(1370)$ as unequivocal states in the spectrum. Within a $1 / N_{c}$ framework, as $N_{c} \rightarrow \infty$ the $\bar{q} q$ states become stable, while the tetraquarks merge with the meson-meson continuum [23, 24]. As we have already noted the real world of scalars with $N_{c}=3$ is distinct from the $N_{c} \rightarrow \infty$ limit. Thus, it is real world meson-meson scattering, as seen in Fig. 1, with whatever states it encompasses, that determines the $\bar{q} q$ condensate that drives chiral symmetry breaking, and vice versa.

Indeed in a quite different scenario, Minkowski and Ochs [25] have proposed that it is the lightest scalar, the $f_{0}(400-1200)$, that is the glueball (or "red dragon" as they call it). $J / \psi$ decay has often been regarded as a source of glue. Long ago both the Mark III and DM2 experiments found $J / \psi \rightarrow \omega(\pi \pi)$ decay has a low mass $\pi \pi$ enhancement. Statistics were not sufficient to allow a partial wave separation. With the higher event rates now available at BEPC, such a separation becomes possible. This confirms that the effect is indeed due to $S$-wave $\pi \pi$ interactions. BESII have fitted this with Breit-Wigner forms and found [26] $M=(541 \pm 39) \mathrm{MeV}$ and $\Gamma=(504 \pm 84)$ 
MeV. Before this, E791 analysed $D^{ \pm} \rightarrow \pi^{ \pm} \pi^{+} \pi^{-}$decay and found a low mass enhancement too. Their Breit-Wigner fit [27] yields a similar $M=(478 \pm 29) \mathrm{MeV}$ but a narrower $\Gamma=(324 \pm 23)$ $\mathrm{MeV}$. The FOCUS experiment has comparable $D$-decay data and a similar fit reveals a $\sigma$ with similar parameters. As emphasised earlier the scalars seen in Fig. 1 are inextricably linked, yet the E791 fit [28] to $D_{s} \rightarrow 3 \pi$ introduces a Breit-Wigner description for a state at $1434 \mathrm{MeV}$ with width of $173 \mathrm{MeV}$, which is neither the $f_{0}(1370)$ or $f_{0}(1500)$, seen in Fig. 1. Similarly, Belle fit $\bar{D}^{0} \rightarrow K_{s} \pi^{+} \pi^{-}$with not only a low mass $\sigma$, but another near $1 \mathrm{GeV}$ in addition to the $f_{0}(980)$ [29] - again not known in $\pi \pi$ scattering. However, FOCUS [30] have also analysed their data on $D \rightarrow 3 \pi$ in a way consistent with the $\pi \pi$ scattering results shown in Fig. 1 and found an even better fit. This does not mean that a low mass $\sigma$ is not needed, only that the $\pi \pi$ final state interactions are consistent with elastic scattering. Such a broad bump is not necessarily resonant, even if it can be fitted by a Breit-Wigner form! One has to show that there is indeed the corresponding phase variation. For the FOCUS data tests of this are still going on. However, for the BESII results on $J / \psi$ decay, Bugg has analysed these in Ref. 31. Using the interference with the crossed bands of $\omega \pi$ interactions in both $S$ and $D$-waves that produce the $b_{1}$, he has shown that the $\pi \pi S$-wave has a phase variation totally consistent with results on $\pi \pi$ scattering, Fig. 4 . As the parametrisation of this by Anisovich and Sarantsev [32], for instance, illustrates perfectly, this does not require a low mass pole in the complex plane.

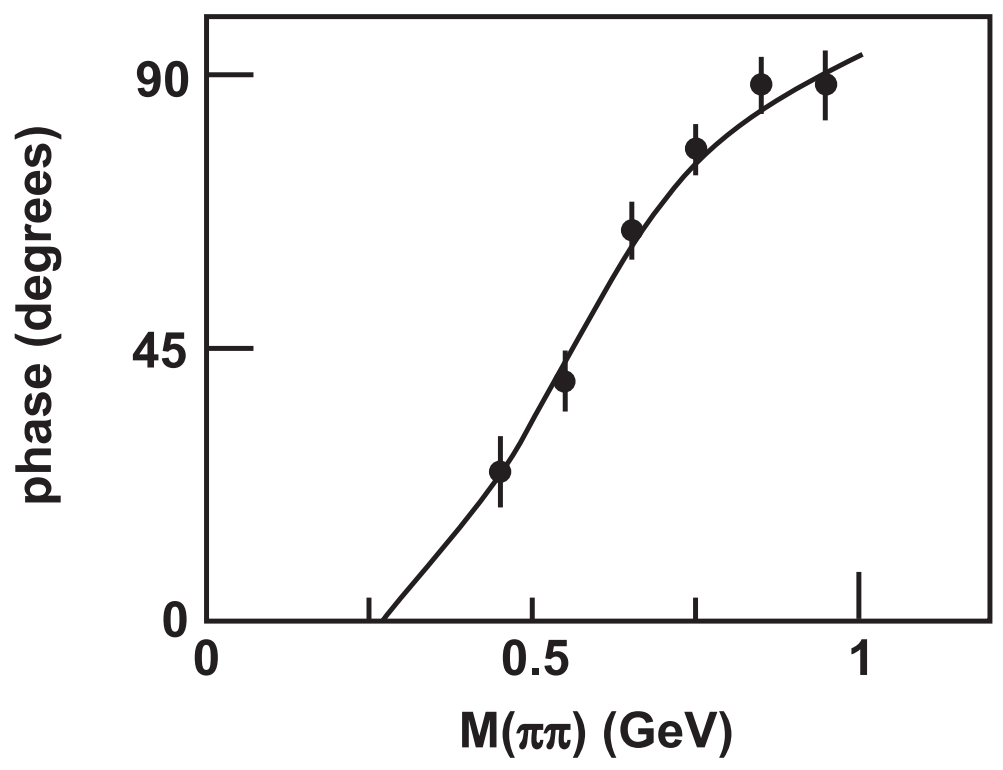

Figure 4: Phase of the $\pi \pi S$-wave in $J / \psi \rightarrow \omega(\pi \pi)$ determined from simultaneous fitting of the magnitudes and phases in each bin from Bugg ${ }^{31}$.

Of course, simple Breit-Wigner forms for such a low mass enhancement presuppose a pole in the complex $s$-plane whether it is there or not. Moreover, they do not incorporate the correct analytic structure along the nearby left hand cut (just like Fig. 2 for $K \pi$ scattering), but for $\pi \pi$ scattering this is further constrained by three channel crossing symmetry. Fits by Zhou et al. [33] implementing crossing sum-rules find a pole at $M=(470 \pm 50) \mathrm{MeV}$ with $\Gamma=(570 \pm 50)$ $\mathrm{MeV}$. This compares very closely to the results of Colangelo, Gasser and Leutwyler [34]. Their 
implementation of the Roy equations determines near threshold $\pi \pi$ scattering as precisely as is presently possible. The $I=0 S$-wave is fitted with a parametrisation due to Schenk [35, 34]. When continued into the complex plane this gives a pole within the region found by Zhou et al. [33]. However, a pole so far from the real axis is not so readily determined. Unitarisations of chiral perturbation theory, like the inverse amplitude method, do find a low mass pole, as reviewed some time ago in Ref. 36, but once again this does not prove that such methods provide the correct amplitude on the unphysical sheet. More work is needed to prove that this is not just the result of using too simplistic representations. We are however reaching a situation that precision data on heavy flavour decays when combined with correct analytic representations will determine whether the $\kappa$ and $\sigma$ (and $f_{0}(1370)$ for that matter) really exist as mesons in the spectrum of nature or not. Claims of states quoted above which differ in width by hundreds of $\mathrm{MeV}$ depending on the representation used, do not yet prove that the true pole may not yet be further away and even off at infinity. The fields that are the Higgs of the strong interaction must remain an enigma for a little longer.

\section{Acknowledgments}

It is a pleasure to thank Professors Kuang-Ta Chao and Chuan Li and their colleagues for the invitation to give this talk and to Bing-Song Zou, Ai-lin Zhang and Qiang Zhao for their very kind hospitality. I acknowledge partial support of the EU-RTN Programme, Contract No. HPRN-CT2002-00311, "EURIDICE".

\section{References}

[1] S. Eidelman et al. [PDG], Phys. Lett. B592 (2004) 1.

[2] Y. Nambu, Phys. Rev. Lett. 4 (1960) 380; Y. Nambu and G. Jona-Lasinio, Phys. Rev. 122 (1960) 345, 124 (1960) 246; M. Gell-Mann and M. Levy, Nuovo Cim. 16 (1960) 705.

[3] M.R. Pennington, Swimming with Quarks, lectures given at the XIth Mexican School of Particles \& Fields, Xalapa, 2004 (to be published) [hep-ph/0504262] (and references therein).

[4] S. Pislak et al. [BNL-E865], Phys. Rev. Lett. 87 (2001) 221801.

[5] B. Adeva et al. [DIRAC Collaboration], Phys. Lett. B 619 (2005) 50 [hep-ex/0504044].

[6] G. Colangelo, J. Gasser and H. Leutwyler, Phys. Rev. Lett. 86 (2001) 5008 [hep-ph/0103063].

[7] M. Gell-Mann, R.J. Oakes and B. Renner, Phys. Rev. 175 (1968) 2195.

[8] D. Aston et al. [LASS], Nucl. Phys. B296 (1988) 493.

[9] D. Alde et al. [GAMS], Phys. Lett. 205B (1988) 397.

[10] B.S. Zou, hep-ph/9611235. Talk presented at 34th Course of International School of Subnuclear Physics, Erice, Sicily, July 1996; V.V. Anisovich, D.V. Bugg, A.V. Sarantsev, B.S. Zou, Phys.Rev. D50 (1994) 972; D.V. Bugg, B.S. Zou, A.V. Sarantsev, Nucl. Phys. B471 (1996) 59.

[11] S. N. Cherry and M. R. Pennington, Nucl. Phys. A688 (2001) 823 [hep-ph/0005208].

[12] J. M. Link et al. [FOCUS], Phys. Lett. B535 (2002) 43 [hep-ex/0203031]. 
[13] B. T. Meadows, Talk at 40th Rencontres de Moriond on QCD and High Energy Hadronic Interactions, La Thuile, Aosta Valley, Italy, 12-19 Mar 2005, [hep-ex/0506040].

[14] L. Edera and M. R. Pennington, Phys. Lett. B623 (2005) 55 [hep-ph/0506117].

[15] K. L. Au, D. Morgan and M. R. Pennington, Phys. Rev. D35 (1987) 1633.

[16] J. D. Weinstein and N. Isgur, Phys. Rev. D41 (1990) 2236.

[17] E. Van Beveren et al., Z. Phys. C30 (1986) 615; N. A. Tornqvist, Z. Phys. C68 (1995) 647; M. Boglione and M. R. Pennington, Phys. Rev. Lett. 79 (1997) 1998 [hep-ph/9703257].

[18] P. Geiger and N. Isgur, Phys. Rev. D47 (1993) 5050; S. Descotes and J. Stern, Phys. Lett. B488 (2000) 274 [hep-ph/0007082]; S. Descotes, JHEP 0103 (2001) 002 [hep-ph/0012221].

[19] R. L. Jaffe, Phys. Rev. D15 (1977) 267; R. L. Jaffe and F. Wilczek, Phys. Rev. Lett. 91 (2003) 232003 [hep-ph/0307341].

[20] D. Black, A. H. Fariborz, F. Sannino and J. Schechter, Phys. Rev. D59 (1999) 074026 [hep-ph/9808415]; D. Black, A. H. Fariborz and J. Schechter, Phys. Rev. D61 (2000) 074001 [hep-ph/9907516].

[21] L. Maiani, F. Piccinini, A. D. Polosa and V. Riquer, Phys. Rev. Lett. 93 (2004) 212002 [hep-ph/0407017].

[22] F. E. Close and Q. Zhao, Phys. Rev. D71 (2005) 094022 [hep-ph/0504043].

[23] R L. Jaffe, e.g. in lectures at the LNF Spring School, Frascati (May 2005).

[24] J. R. Pelaez, Mod. Phys. Lett A19 (2004) 2879 [hep-ph/0411107].

[25] P. Minkowski and W. Ochs, Eur. Phys. J. C9 (1999) 283 [hep-ph/9811518].

[26] M. Ablikim et al. [BES II], Phys. Lett. B598 (2004) 149 [hep-ex/0406038].

[27] E.M. Aitala et al. [Fermilab E791], Phys. Rev. Lett. 86 (2001) 770 [hep-ex/0007028].

[28] E.M. Aitala et al. [Fermilab E791], Phys. Rev. Lett. 86 (2001) 765 [hep-ex/0007027].

[29] A. Poluektov et al. [Belle], Phys. Rev. D70 (2004) 072003 [hep-ex/0406067].

[30] S. Malvezzi, "Light quark and charm interplay in the Dalitz-plot analysis of hadronic decays in FOCUS," [hep-ex/0307055]; J. M. Link et al. [FOCUS], Phys. Lett. B585 (2004) 200 [hep-ex/0312040].

[31] D. V. Bugg, Eur. Phys. J. C37 (2004) 433 [hep-ex/0411042].

[32] V.V. Anisovich and A.V. Sarantsev, Eur. Phys. J. A16 (2003) 229 [hep-ph/0204328].

[33] Z. Y. Zhou, G. Y. Qin, P. Zhang, Z. G. Xiao, H. Q. Zheng and N. Wu, JHEP 0502 (2005) 043 [hep-ph/0406271].

[34] G. Colangelo, J. Gasser and H. Leutwyler, Nucl. Phys. B603 (2001) 125 [hep-ph/0103088].

[35] A. Schenk, Nucl. Phys. B363 (1991) 97.

[36] M.R. Pennington, "Riddle of the scalars: where is the $\sigma$ ?", Proc. Int. Workshop on Hadron Spectroscopy, Frascati, 1999 (ed. T. Bressani et al.) (pub. Frascati Physics Series, Vol. 15) pp. $95-114$ [hep-ph/9905241]. 